\title{
Propolis Protective Effects Against Doxorubicin-Induced Multi-Organ Toxicity via Suppression of Oxidative Stress, Inflammation, Apoptosis, and Histopathological Alterations in Female Albino Rats
}

\author{
Ehsan Khedre Mohamed 1(D), Amany Abdelhameid Osman 2 (D), Asmaa M. Moghazy ${ }^{3}$ (D), Amina A.S. \\ Abdel Rahman $2, *$ iD \\ 1 Biochemistry department, National Organization of Drug Control and Research (NODCAR), Giza, Egypt \\ drehsankhedre@hotmail.com (E.K.M.); \\ 2 Department of Zoology, Faculty of Women for Arts, Science and Education, Ain shams university, Cairo, Egypt; \\ Amanyosman@women.asu.edu.eg (A.A.O); amina_abdelrahman@women.asu.edu.eg (A.A.S.A.R); \\ 3 Hormones Department, NODCAR, Giza, Egypt; asmaamoghazy@ hotmail.com (A.M.M.); \\ * Correspondence: amina_abdelrahman@women.asu.edu.eg (A.A.S.A.R.);
}

Scopus Author ID 57207827047

Received: 15.04.2021; Revised: 16.05.2021; Accepted: 20.05.2021; Published: 10.06.2021;

\begin{abstract}
Doxorubicin (DOX) is effective chemotherapy in several malignancies, but large-scale toxicities limit its clinical usefulness. Propolis has been reported to exhibit a broad spectrum of biological activities. We aim to assess the protective efficacy of propolis against DOX-induced multitoxicity in female rats. Forty female rats were divided into four groups: control group; Group (P) were administrated oral propolis (100 mg/kg once daily for 28 days); Group (P+DOX) were injected with a single intraperitoneal dose of DOX $\left(20 \mathrm{mg} / \mathrm{kg}\right.$ i.p at $24^{\text {th }}$ day after the propolis administration $)$ and group (DOX) were injected with doxorubicin only. Estimation of cardiac, renal and hepatic injury markers, apoptosis and pro-inflammatory cytokines were done using sera. Also, liver and heart tissue samples were collected to determine GSH and MDA as oxidative stress markers. In addition to histopathological and immunohistochemical examination of Cytochrome-C and Connexin43 on lysed myocardium, liver, kidney and lung tissues. Doxorubicin toxicity caused marked deteriorations of measured parameters through the different mechanisms in different body organs. However, pre-treatment with propolis significantly ameliorated these alterations. Thus propolis can ameliorate the DOX-induced experimental multi-toxicity as cardiomyopathy, hepatotoxicity, nephritis and pneumonia. Thus, it could be a promising protective agent in DOX treatment protocols.
\end{abstract}

Keywords: Propolis, Cytochrome-C, Caspase-3, Connexin 43, Doxorubicin, Interleukin-1 $\beta$, Multitoxicity.

(C) 2021 by the authors. This article is an open-access article distributed under the terms and conditions of the Creative Commons Attribution (CC BY) license (https://creativecommons.org/licenses/by/4.0/).

\section{Introduction}

Doxorubicin (DOX) or Adriamycin ${ }^{\circledR}$ is an active chemotherapeutic drug used in several malignancies like breast, ovarian, lung and uterine cancers. However, its clinical usefulness is limited by large-scale toxicities, including cardiac, hepatic, renal, pulmonary, hematological, and testicular toxicity [1]. One way to overcome these severe side effects is to figure out the mechanism of its adverse effects. Many strategies have been tried to minimize these side effects by adding a protective agent to DOX treatment protocols. Some of the 
suggested mechanisms of DOX toxicities are oxidative stress, inflammation, endoplasmic reticulum-mediated apoptosis, and DNA/RNA damage. The metabolism of DOX by cytochrome P-450 enzyme leads to the formation of hydroxyl radicals and superoxide anions, causing cellular membrane injury [2]. Based on these perceptions, various antioxidants, antiinflammatory or anti-apoptotic agents have been recommended to oppose DOX-induced damages. Nevertheless, no single treatment has proven effective enough to reverse or even prevent these adverse effects.

Propolis is a sticky substance collected by honeybees (Apis mellifera L.) from various plants. According to its source, geographical, climate, and age, the contents and efficiency of propolis vary greatly. It contains more than 150 polyphenol compounds such as flavonoids and phenolic acid, including their esters. However, the mechanism of action of these flavonoids is not well-defined. It also contains vitamins $\mathrm{E}$ and $\mathrm{C}$; vitamin $\mathrm{B}$ complexes, some trace elements and several essential minerals such as $\mathrm{Ca}, \mathrm{Zn}, \mathrm{Mg}, \mathrm{Cu}, \mathrm{Mn}, \mathrm{Fe}$, and Ni [3]. Thus, it exhibits various biological activities as free radical scavenging, antioxidant, cytotoxic, and antimicrobial effects [4]. As well, its anti-inflammatory, antiviral, and immunomodulatory effects were reported, along with perspectives for its using for coronavirus 2019 (COVID-19) [5]. Keeping this information in mind, we aim in our study to determine whether propolis could attenuate DOX-induced apoptosis and oxidative stress and thus its serious side effects. This evaluation included the biochemical, histopathological, and immunohistochemical changes in all-female rats to investigate propolis' mechanism in vivo as an antioxidant, anti-inflammatory, and anti-apoptotic agent.

\section{Materials and Methods}

\subsection{Chemicals.}

DOX vials (Doxorubicin Ebewe, Ebewe Pharma GmbH, Unterach, Austria). Propolis dry extract from Healthy Care Propolis 2000 mg, 200 Capsules (Australia Import ). All other chemicals and reagents used were of analytical grade.

\subsection{Phytochemical analysis of propolis capsules.}

\subsubsection{High-performance liquid chromatography (HPLC).}

Flavonoids and other phenolic acids in propolis capsules were detected and quantified using diode array detection at 280, 320, and $360 \mathrm{~nm}$ against standards, Eclipse XDB C18 column (150 x 4.6 um 5 um Agilent technologies 1100 series with autosampler [6].

\subsubsection{DPPH assay.}

The radical scavenging capacity of propolis was measured by bleaching the purple color of 1,1-diphenyl-2-picrylhydrazyl radical (DPPH) solution according to a previous method [7]. $1 \mathrm{~mL}$ from propolis' different concentrations $(50 \mu \mathrm{g} / \mathrm{mL}, 25 \mu \mathrm{g} / \mathrm{mL}$ and $10 \mu \mathrm{g} / \mathrm{mL})$ was combined to $0.5 \mathrm{~mL}$ of a $0.2 \mathrm{mmol} / \mathrm{L} \mathrm{DPPH}$ methanolic solution. The absorbance was measured at $517 \mathrm{~nm}$ after keeping in the dark for 30 minutes. $\mathrm{IC}_{50}(\mu \mathrm{g} / \mathrm{mL})$, the capability to scavenge $50 \%$ of the DPPH radical concentration, was calculated as compared to $\alpha$-Tocopherol from the equation: scavenging effect of $\mathrm{DPPH}=\left[\left(\mathrm{A}_{0}-\mathrm{A}_{1}\right) / \mathrm{A}_{0}\right] * 100 . \mathrm{A}_{0}$ is the absorbance of the control, and $\mathrm{A}_{1}$ is the absorbance of the sample. 


\subsection{Animals.}

Forty healthy female albino rats weighing $190 \pm 10 \mathrm{~g}$ were provided by the breeding unit of the National Organization for Drug Control and Research (NODCAR), Giza, Egypt. Rats were housed under controlled conditions $\left(21 \pm 1^{\circ} \mathrm{C}\right.$ constant temperature, humidity $55 \%$, $12 \mathrm{~h}$ light-dark cycle), and received standard diet and water ad libitum. The study complied with the Guide for Care and Use of Laboratory Animals issued by the US National Institutes of Health (NIH Publication No. 8023, revised 1978) and was approved by NODCAR Research Ethics Committee (REC-Protocol Number: NODCAR II 57 19). Animals were maintained for 14 days before the experiment as an acclimatization period.

\subsection{Experimental design.}

Rats were divided into four equal groups randomly (10 animals each); Group I (control group): animals were administrated normal saline. Group II (P): animals were received 100 $\mathrm{mg} / \mathrm{kg}$ body weight of oral propolis for 28 consecutive days to determine the toxic and undesired effects of propolis [3]. Group III (P+DOX): animals were injected with a single intraperitoneal dose of DOX (20 mg/kg i.p [8]) on the $24^{\text {th }}$ day after the propolis administration for 28 consecutive days. Group IV (DOX, positive control): animals have administrated only a single dose of $20 \mathrm{mg} / \mathrm{kg}$ DOX i.p. on the $24^{\text {th }}$ day.

All the animals were sacrificed on day $28^{\text {th }}$ by decapitation. Blood samples have been collected from cardiac puncture and the sera were separated and kept at $-20{ }^{\circ} \mathrm{C}$ for further analysis. Heart and liver tissue samples were collected to determine oxidative stress markers and heart, kidney, lung, and liver tissue samples for histopathological and immunohistochemical examinations.

\subsection{Determination of relative body weight $(R B W)$.}

Relative body weight was calculated as the difference between the final weight and the initial weight divided by the initial weight.

\subsection{Biochemical analysis.}

2.6.1. Measurement of serum biomarkers.

The collected sera were used to determine creatine kinase (CK), creatine kinasemyocardial B fraction (CK-MB), lactate dehydrogenase (LDH), alanine aminotransferase (ALT), aspartate aminotransferase (AST), alkaline phosphatase (ALP), triglyceride (TG), total cholesterol (TC), blood urea nitrogen (BUN), uric acid, creatinine, albumin, and total protein. All the previous biochemical tests were done using commercial kits of Human, Biosystem, Spectrum, and Biomed diagnostic Inc. Serum interleukin-1 $\beta$ (IL-1 $\beta$ ) and Caspase-3 were assayed using rat ELISA kits supplied by Bioassay Technology Laboratory.

\subsubsection{Oxidative stress and antioxidant activity.}

Liver tissues were homogenized in chilled $10 \mathrm{mM}$ PBS with optimal $\mathrm{pH} 7.4$ and were used to measure malondialdehyde (MDA) using the thiobarbituric acid test [9] and glutathione (GSH) content [10] by HPLC method. 


\subsection{Histopathological and immunohistochemical examination.}

Hematoxylin and eosin (H\&E) stain was performed on formalin-fixed, paraffinembedded tissue samples ( heart, liver, kidney, and lung) according to a method described previously [11]. Deparaffinized tissue sections were specifically stained immunohistochemically for detection of mouse monoclonal anti-animal connexin 43(Cx43) antibody (1: 400 dilution, 3512, Cell Signaling Technology, Danvers, M, US) and monoclonal mouse anti-cytochrome C (Cyt-C) (1:1000 dilution, Zymed Laboratories, San Francisco, CA) [12]. After incubations, tissue sections were washed by saline and incubated with diaminobenzidine for $10 \mathrm{~min}$, then counterstained with hematoxylin. The percentage of immune-reactive cells and the overall area tissue cells were calculated and statistically analyzed using color extraction software (A TinEye Lab powered by MulticolorEngine https://labs.tineye.com/color/).

\subsection{Statistical analysis.}

Data are expressed as mean \pm SE. Comparisons between different groups were made using one-way analysis (ANOVA) and Tukey's multiple comparisons test using the software GraphPad InStat and Statistical Package for the Social Sciences program (SPSS version 20.0, Chicago, USA). A probability level of less than 0.05 was accepted as statistically significant [13].

\section{Results}

\subsection{HPLC chromatograms of the phenolic fractions of propolis.}

The phenolic compounds and flavonoids are of major constituents found in propolis when compared to standards. Among the identified compounds, rosmarinic acid (caffeic acid ester), sinapic acid (hydroxycinnamic acid), and apigenin (4',5,7-trihydroxyflavone) were the most abundant compounds. Also, cinnamic, ferulic, and caffeic acids, as well as quercetin and kaempferol, showed considerable amounts (Table 1). Unexpectedly, gallic, protocatechuic, phydroxybenzoic, gentisic, chlorogenic, syringic, vanillic, $p$-coumaric acids, catechin, rutin, apigenin-7-glucoside, and chrysin showed undetectable amounts.

Table 1. Main phenolic compounds of propolis detected by HPLC.

\begin{tabular}{l|c|c}
\multicolumn{1}{c|}{ Compound } & Retention time & Concentration $(\boldsymbol{\mu g} / \mathbf{g})$ \\
\hline Caffeic acid & 17.2 & 58.39 \\
\hline Ferulic acid & 28.7 & 82.66 \\
\hline Sinapic acid & 30.4 & 210.41 \\
\hline Rosmarinic acid & 39.2 & 834.27 \\
\hline Cinnamic acid & 44.9 & 111.13 \\
\hline Qurecetin & 48.7 & 11.32 \\
\hline Apigenin & 54.2 & 159.76 \\
\hline Kaempferol & 54.9 & 36.24
\end{tabular}

\subsection{Scavenging activity of propolis.}

One of the more prominent properties of flavonoids is their excellent radical scavenging ability. Compared to vitamin E, propolis showed a high scavenging effect. Propolis reduced free radical DPPH in a concentration-dependent manner. At $50 \mu \mathrm{g} / \mathrm{mL}$, propolis ethanolic extract reduces $\mathrm{DPPH}^{\circ}$ by $42.5 \%$ against $98.1 \%$ observed with Vit. E (Table 2). The $\mathrm{IC}_{50}$ for antioxidant activity of propolis and Vit $\mathrm{E}$ are $70 \mu \mathrm{g} / \mathrm{mL}$ and $17.1 \mu \mathrm{g} / \mathrm{mL}$, respectively. 
Table 2. Scavenging effect and $\mathrm{LC}_{50}$ of the propolis ethanolic extract and Vit. E against $\mathrm{DPPH}^{\circ}$ free radicals.

\begin{tabular}{c|c|c|c|c} 
& $10 \mu \mathrm{g} / \mathrm{mL}$ & $25 \mu \mathrm{g} / \mathrm{mL}$ & $50 \mu \mathrm{g} / \mathrm{mL}$ & $\mathrm{LC} 50 \mu \mathrm{g} / \mathrm{mL}$ \\
\hline$\alpha$-Tocopherol & 31.0 & 74.8 & 98.1 & 17.1 \\
\hline Propolis & 27.6 & 33.5 & 42.5 & 70.0
\end{tabular}

\subsection{Effects on cardiac markers.}

The level of both serum CK and CK-MB were significantly $(\mathrm{P}<0.001)$ increased by about 1.4 and 2-folds, respectively, in DOX-treated animals versus the control group (Figure $1 \mathrm{~A} \& \mathrm{~B})$. However, $\mathrm{CK}$ level was significantly $(\mathrm{P}<0.001)$ reduced by $26 \%$ when the animals were concomitantly treated with propolis. Propolis, prophylactically administered 24 days before DOX, was more effective in lowering the CK-MB level than total CK by $51 \%$ as compared to DOX-treated animals. Moreover, DOX significantly $(\mathrm{P}<0.001)$ increased serum LDH, TC, and TG levels by 1.7, 1.2 and 1.3 folds, respectively, versus the control group (Figure $1 \mathrm{C}-\mathrm{E})$. On the contrary, pre-treatment with propolis significantly $(\mathrm{P}<0.001)$ reduced DOXinduced changes by $40 \%, 17 \%$, and $24 \%$, respectively, compared to the untreated DOX group.

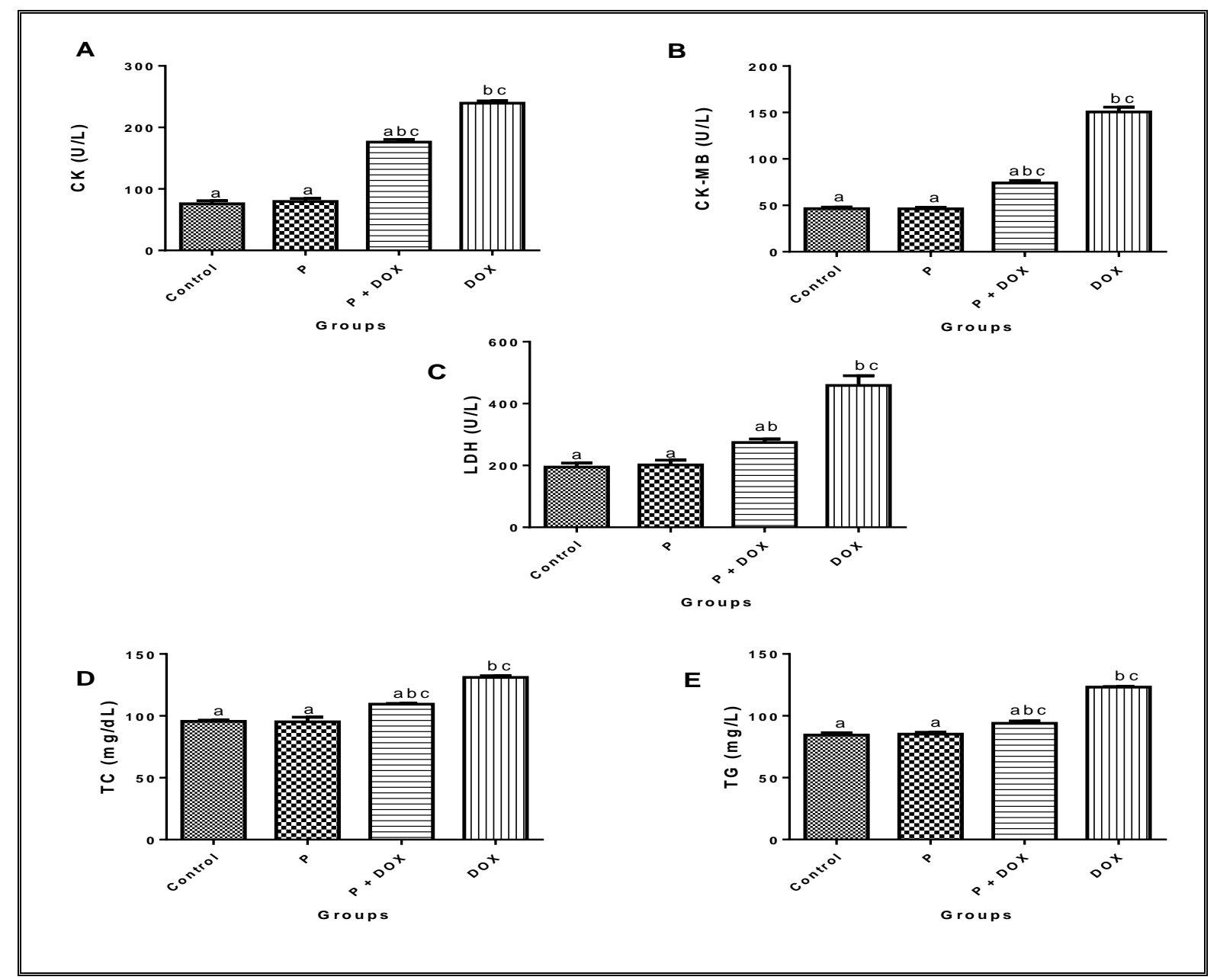

Figure 1. Effect of a single dose of doxorubicin $(20 \mathrm{mg} / \mathrm{kg})$ alone or combined with propolis $(100 \mathrm{mg} / \mathrm{kg}) \mathrm{on}$ cardiac markers. Groups: Control, Propolis (P), P+ DOX, DOX. Data are expressed as mean \pm SE $(n=10){ }^{a}$ Significantly different from the DOX group at $\mathrm{p}<0.05,{ }^{\mathrm{b}}$ Significantly different from the control group at $\mathrm{p}<$ 0.05 and ${ }^{\mathrm{c}}$ Significantly different from the propolis group.

\subsection{Effects on hepatic markers.}

Parallel to the observed increase in cardiac markers, DOX significantly $(\mathrm{P}<0.001)$ increased liver dysfunction serum indices, including AST, ALT, and ALP activities by 1.5, 1.6, 
and 1.5-folds compared to control animals. Treatment of rats by propolis attenuated DOXinduced changes in the parameters via $32 \%, 38 \%$, and $32 \%$, respectively, versus the DOX group (Figure $2 \mathrm{~A}-\mathrm{C}$ ). Also, our study stated that DOX induction resulted in a marked decrease $(\mathrm{P}<0.001)$ in both serum albumin and total protein levels by 1.44 and 1.4-folds, respectively, compared to the control group. The only change in total serum protein was largely attenuated $(P<0.05)$ in the propolis group, while administration of propolis could not prevent DOXinduced increases in serum levels of albumin significantly $(\mathrm{P}=0.051)$ when compared to the DOX group (Figure 2 D\&E).

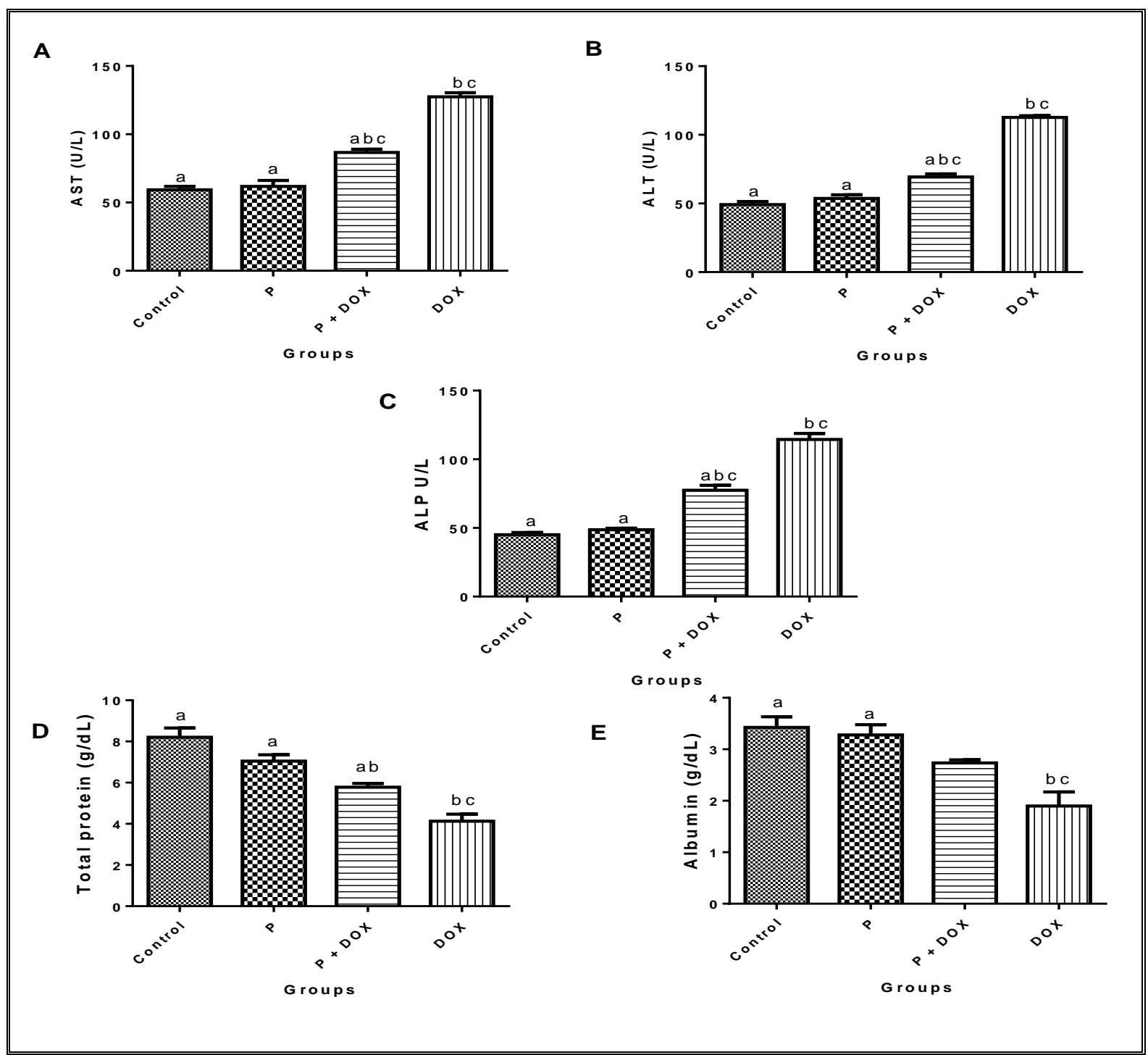

Figure 2. Effect of a single dose of doxorubicin $(20 \mathrm{mg} / \mathrm{kg}$ ) alone or combined with propolis $(100 \mathrm{mg} / \mathrm{kg}) \mathrm{on}$ hepatic markers. Groups: Control, Propolis $(\mathrm{P}), \mathrm{P}+\mathrm{DOX}, \mathrm{DOX}$. Data are expressed as mean \pm SE $(\mathrm{n}=10){ }^{\mathrm{a}}$ Significantly different from the DOX group at $\mathrm{p}<0.05,{ }^{\mathrm{b}}$ Significantly different from the normal control group at $\mathrm{p}<0.05$ and ${ }^{\mathrm{c}}$ Significantly different from the propolis group.

\subsection{Effect on renal biomarkers.}

Moreover, DOX significantly $(\mathrm{P}<0.001)$ increased serum indices of renal dysfunction including BUN, creatinine, and uric acid by 1.5, 2 and 2.3-folds, respectively as compared to the normal control group, in addition to albumin and total protein mentioned before. In contrast, pre-treatment with propolis significantly $(\mathrm{P}<0.001)$ reduced DOX-induced changes by $33 \%$, $50 \%$ and $56 \%$, respectively, if compared to the untreated DOX group, as shown in Figure 3. 
A

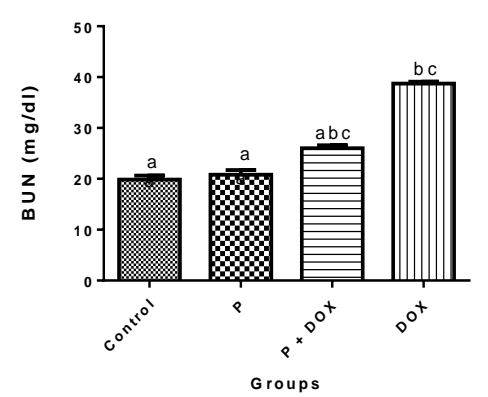

B

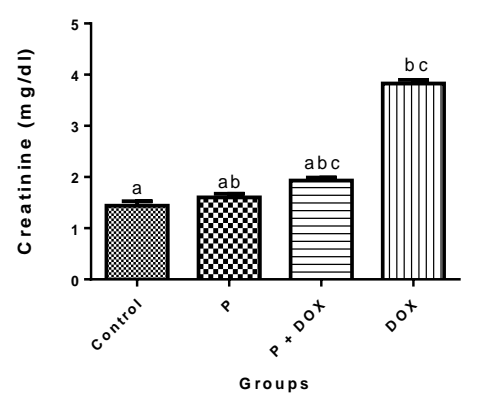

C

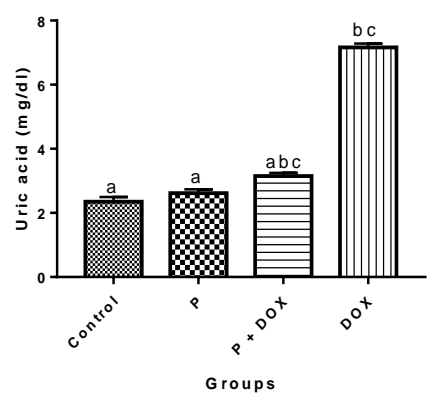

Figure 3. Effect of a single dose of doxorubicin $(20 \mathrm{mg} / \mathrm{kg})$ alone or combined with propolis $(100 \mathrm{mg} / \mathrm{kg})$ on renal markers. Groups: Control, Propolis (P), P+DOX, DOX. Data are expressed as mean $\pm \mathrm{SE}(\mathrm{n}=10)$. ${ }^{\mathrm{a}}$ Significantly different from the DOX group at $\mathrm{p}<0.05,{ }^{\mathrm{b}}$ Significantly different from the control group at $\mathrm{p}<$ 0.05 and ${ }^{\mathrm{c}}$ Significantly different from the propolis group.

\subsection{Effect on oxidative stress.}

Regarding the oxidative markers, Dox significantly $(\mathrm{P}<0.001)$ increased the MDA content of both heart and liver tissues by 1.43 and 1.42-folds, respectively, versus control animals. Though propolis treatment attenuated DOX effects only in the heart via significant $(\mathrm{P}<0.001)$, decreasing the MDA content by $11.8 \%$ versus untreated DOX group but could not prevent DOX effect on liver significantly $(\mathrm{P}=0.054)$ when compared to untreated DOX group (Fig. 5A\&B). Also, DOX caused a marked decrease $(\mathrm{P}<0.001)$ in GSH content of both heart and liver tissues by 1.21 and 1.18-folds, respectively, compared with the control group. However, the propolis pre-treatment increased GSH content significantly $(\mathrm{P}=0.0167$ and 0.0161 ) by $7.4 \%$ and $9.2 \%$ in heart and liver tissues, respectively, compared with the untreated DOX group (Figure. $4 \mathrm{~A} \& \mathrm{~B})$.

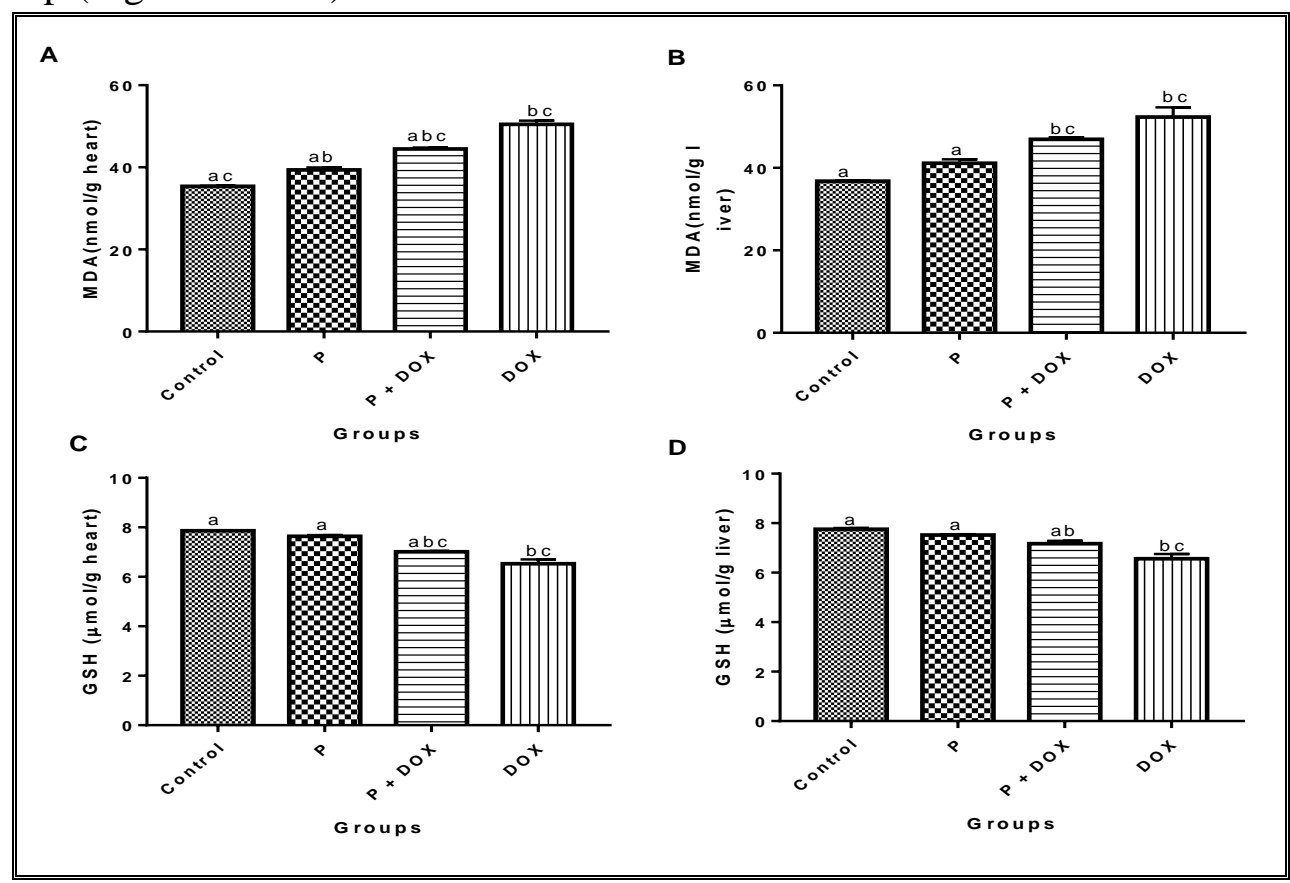

Figure 4. Effect of a single dose of doxorubicin $(20 \mathrm{mg} / \mathrm{kg}$ ) alone or combined with propolis $(100 \mathrm{mg} / \mathrm{kg})$ on oxidative stress markers A\&B) MDA and C\&D) GSH in heart and liver, respectively. Groups: Control, Propolis $(\mathrm{P}), \mathrm{P}+\mathrm{DOX}, \mathrm{DOX}$. Data are expressed as mean $\pm \mathrm{SE}(\mathrm{n}=10) .{ }^{\mathrm{a}}$ Significantly different from the DOX group at $\mathrm{p}<0.05,{ }^{\mathrm{b}}$ Significantly different from the control group at $\mathrm{p}<0.05$ and ${ }^{\mathrm{c}}$ Significantly different from the 


\subsection{Body Weight change and effects on inflammatory and apoptotic biomarkers.}

Our study revealed that the RBW was significantly decreased $(\mathrm{P}<0.001)$ by 1.3 -folds in untreated DOX rats than control rats. On the other hand, the propolis prophylactically administrated before Dox exhibited a significant $(\mathrm{P}<0.001)$ increase in RBW by about $26 \%$ versus untreated DOX rats (Figure 5A). Furthermore, data summarized in Fig. 5 B\&C showed that a single dose of DOX increased significantly $(\mathrm{P}<0.001)$ in serum IL- $1 \beta$ and caspase- 3 by 1.4 and 1.6-folds, respectively, the control group. In contrast, administration of propolis produced a significant reduction $(\mathrm{P}<0.01)$ in both markers by $31 \%$ and $39 \%$, respectively.

Our study observed that the administration of propolis to normal rats showed an insignificant difference from the normal control group in all measured parameters.

A

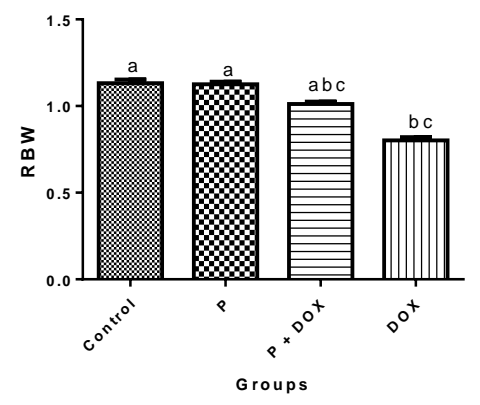

B

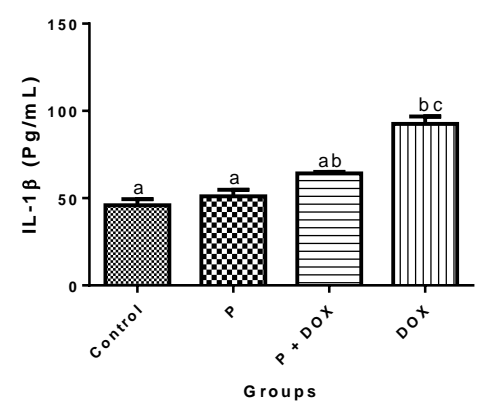

C

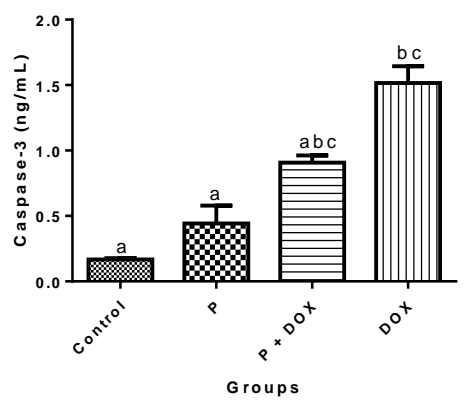

Figure 5. Effect of a single dose of doxorubicin $(20 \mathrm{mg} / \mathrm{kg})$ alone or combined with propolis $(100 \mathrm{mg} / \mathrm{kg})$ on $\mathrm{A})$ RBW, B) IL-1 $\beta$ and C) Caspase-3 levels in the blood. Groups: Control, Propolis (P), P+ DOX, DOX. Data are

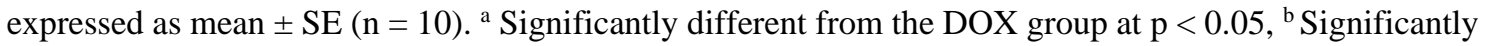
different from the control group at $\mathrm{p}<0.05$, and ${ }^{\mathrm{c}}$ Significantly different from the propolis group.

\subsection{The histopathological examination.}

The current study showed the effect of DOX broad toxicity on the typical structure of various examined tissues of female albino rats with different pathological degrees, as shown in Figure 6.

\subsubsection{Cardiac muscle.}

The cardiomyocytes normally contain one or two fusiform nuclei, the cells are rich in mitochondria as well as actin and myosin proteins arranged in a repeating unit called a sarcomere, intercalated disc and vascularized network of capillaries running in between the fibers, as shown in section $\mathrm{A}$ and $\mathrm{B}$ of the heart of the control group and propolis group (P) alone, respectively. While section $\mathrm{C}$ of $(\mathrm{P}+\mathrm{DOX})$ treated group exhibited slight cardiac muscle necrosis and fibrotic blood vessels. Section D of the DOX group displayed congestive hemorrhage, apoptotic body, fibers depress in the degenerative necrotic area and lipofuscin pigments in damaged parts and hypertrophy. All these previous features in section D may point more directly to cardiomyopathy.

\subsubsection{Hepatic tissue.}

The normal histological structure, as shown in sections A and B of the liver of the control group and propolis group $(\mathrm{P})$ alone, respectively is consisted of the hexagonal hepatic 
lobes arranged as hepatocyte cords and central vein running blood sinusoid contains Kupffer cell and portal triad area. Hepatic tissue of $(\mathrm{P}+\mathrm{DOX})$ group manifested only fatty vacuoles, hemorrhage, necrotic area, and pyknotic nucleus as shown in section $\mathrm{C}$, while hepatic tissue of DOX group illustrates lymphocytes infiltration, apoptotic body, vacuolar hydropic change, and destroyed portal area with bile duct hyperplasia, fibroid portal vein, inflammation and hemorrhage as (section D).

\subsubsection{Kidney tissue.}

The normal histological structure as shown in nephron sections A and B of control and propolis group (P.) alone consists of glomerulus tuft with Bowman's capsule, proximal and distal convoluted tubules lined by cubical epithelial cell. Section $\mathrm{C}$ of $(\mathrm{P}+\mathrm{DOX})$ group elucidates only destroyed the glomerulus tuft, necrotic tubular area. Though, obvious pathological changes in the kidney tubules of the DOX group are observed as lobulated glomeruli tuft and other atrophy, diminution of Bowman's space, fibroid Bowman's capsule, and other notable destroyed convoluted tubules as illustrated in section D.

\subsubsection{Lung tissue.}

The normal histological structure of lung tissue, as shown in sections A and B of the control and propolis group (P.) alone, consists of arrangement alveolar sacs, bronchioles, and a well-developed pseudostratified epithelial layer (PSEL) with goblet cells. In comparison, section $\mathrm{C}$ of $(\mathrm{P}+\mathrm{DOX})$ group illustrates only bronchioles hyperplasia and blood vessel hypertrophy. Section D of the DOX group shows outer extra mucous secretion as an adaptive reaction in bronchioles proliferation goblet cells, lymphocytic inflammation, fibroid capillary, accumulation of blood block, and arterial wall hypertrophy.

\subsection{Immunohistochemical results.}

Alongside histological investigation of functional impairment and pathological damage, the $\mathrm{Cx} 43$ expressions increased dramatically $(\mathrm{P}<0.001)$ by $4.30,4.60,4.37$, and 3.60folds in the heart, liver, kidney, and lung, respectively compared to the control group. While Cyt-C expressions increased significantly $(\mathrm{P}<0.001)$ by 8.80, 6.09, 9.50, and 10.38 -folds in heart, liver, kidney, and lung, respectively, compared to the control group. On the contrary, propolis administration significantly improved the function of $\mathrm{Cx} 43$ channels by 53.03, 52.63, and $57.78 \%$ in the heart, kidney, and lung, respectively, versus the untreated DOX group, while no significant improvement was noticed in the liver $(\mathrm{P}=0.27)$.

Also, Cyt-C expression was inhibited by propolis pre-treatment by 21.71 and $30.56 \%$ in both heart and lung, respectively, versus the untreated DOX group but could not prevent Dox-induced effect significantly $(\mathrm{P}=0.65$ and 0.052$)$ in both liver and kidney, respectively. Ultimately, the severity of heart, liver, kidney, and lung tissue injury was reduced as propolis is administered before DOX (Figure 7).

\section{Discussion}

DOX is considered to be the most toxic anthracycline on body tissues, despite its wide use in clinical oncology treatment due to its great therapeutic efficacy. Dexrazoxane, the only FDA-approved medicine to treat cardiotoxicity, is associated with difficult availability and 
administration. In addition, there is still no agent approved to stand against other organ toxicities of DOX [14].

In the current study, the harmful effects of DOX on different organs were established biochemically and histologically. Considerable efforts have been made to understand the mechanisms of DOX toxicity and to identify therapies that reduce these adverse responses, such as antioxidants. This study aimed to assess propolis' possible efficacy, which possesses multi-organ protective potential while facing DOX toxic damage. In recent years, propolis has paid great attention as a therapeutic and potential preventive agent due to its various reported benefits plus its safety and cost-effectiveness.

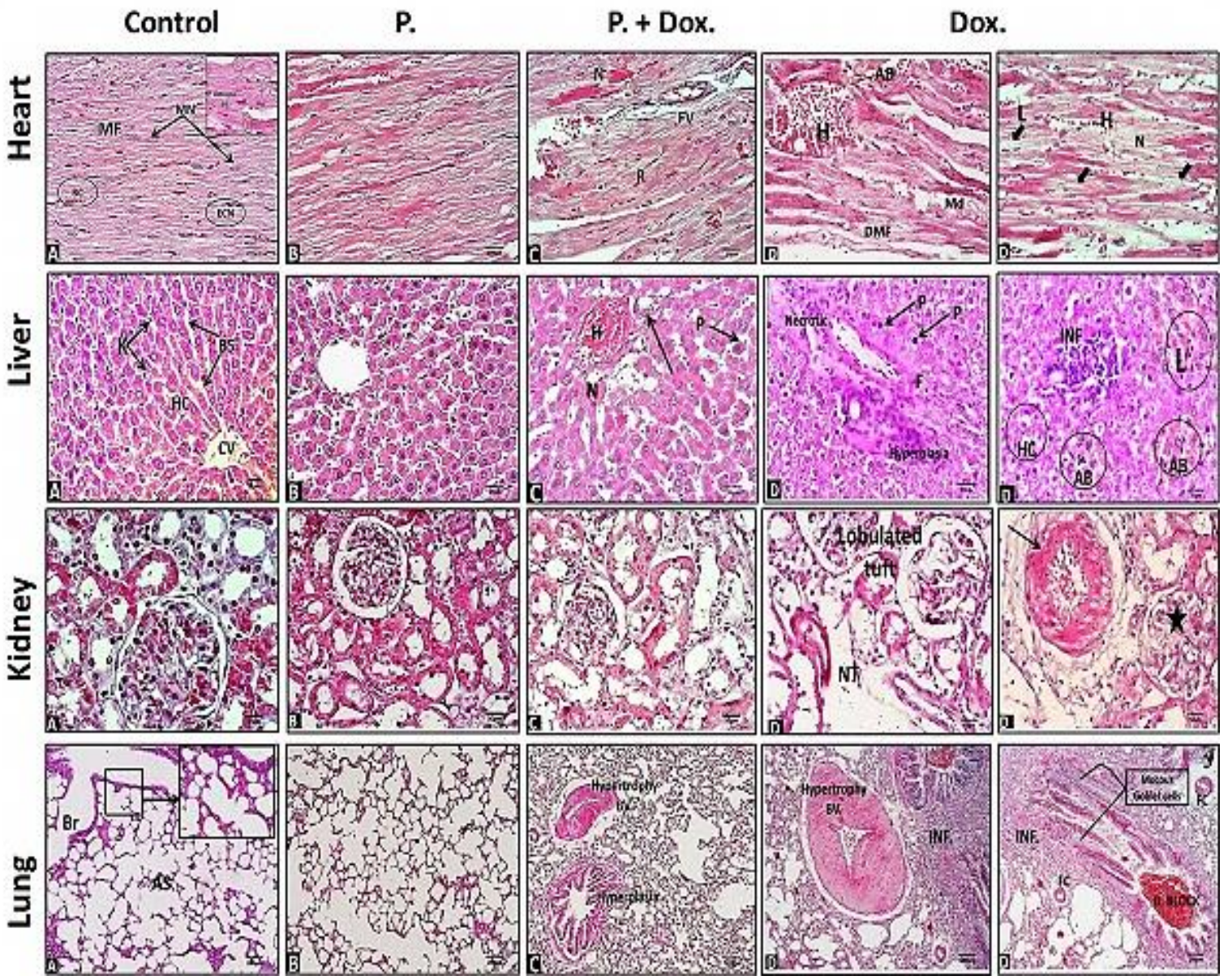

Figure 6. Photomicrograph of tissue sections stained with H\&E, (Heart) Section A: control group shows normal cardiac muscle structure, muscle fibers (MF); muscle nucleus (MN); blood capillary (BC) and Endothelial central nucleus ECN (100, inset $\mathrm{x} 400)$. Section B: propolis treated group showed normal structure. Section $\mathrm{C}:(\mathrm{P}+\mathrm{DOX})$ group shows necrosis $(\mathrm{N})$; fibrotic vessels $(\mathrm{FV})$ and regenerated area (R). Section D: DOX group shows hemorrhage $(\mathrm{H})$, apoptotic body (AB), muscle Fiber depress (MD); degenerative MF (DMF), necrotic area $(\mathrm{N})$, and lipofuscin (L). (Liver $\mathrm{x} 400)$ Section A: normal hepatic tissue where central vein (CV), hepatocyte

(HC); blood sinusoid (BS), and Kupffer cell (K). Section B: propolis treated group shows the normal structure. Section $\mathrm{C}:(\mathrm{P}+\mathrm{DOX})$ treated group illustrates necrotic area $(\mathrm{N})$, hemorrhage $(\mathrm{H})$, and pyknotic nucleus (P). Section D: DOX treated group displays lymphocytes (L), inflammation (INF), apoptotic body (AB), and hydropic change (HC). (Kidney $\mathrm{x} 400$ ) Section A: control group shows normal kidney structure of glomerulus tuft with bowman capsule, nephron tubules. Section B: propolis treated group shows the normal structure. Section C: (P+DOX) treated group showed destroyed tuft cortex tubules. Section D: DOX treated group shows necrotic tubules (NT) and hydropic change, lobulated tuft. (Lung x100) Section A: control group shows normal Lung structure where alveolar sacs (AS), bronchioles (Br), and PSEL, pseudostratified epithelial layer (PSEL). Section B: propolis treated group shows the normal structure. Section C: (P+DOX) treated group illustrates bronchioles hyperplasia and blood vessel hypertrophy. Section D: DOX treated group shows extra mucous secretion in goblet cells, inflammation (INF), fibroid capillary (FC) accumulation of blood block, and blood vessel hypertrophy. Sections $\mathrm{A}=$ control, $\mathrm{B}=\mathrm{P}$ (propolis), $\mathrm{C}=\mathrm{P}+\mathrm{DOX}$ (doxorubicin) and $\mathrm{D}=\mathrm{DOX}$. 
A

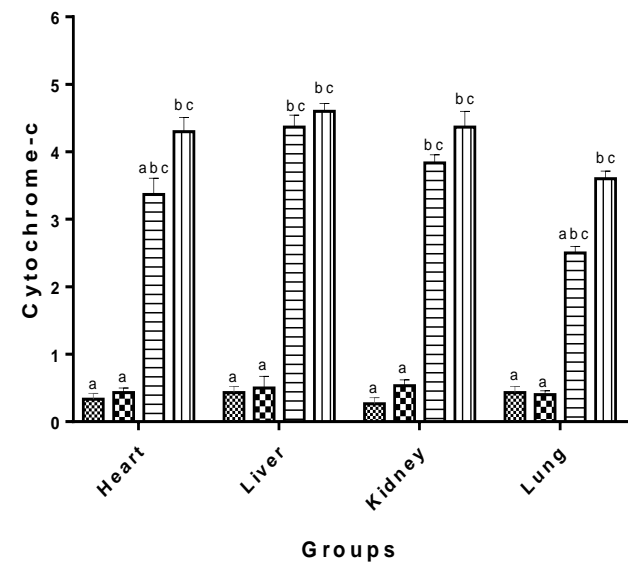

B

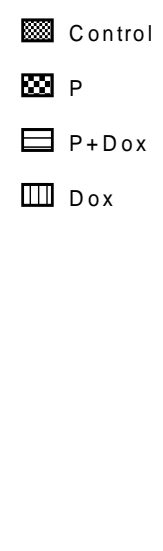

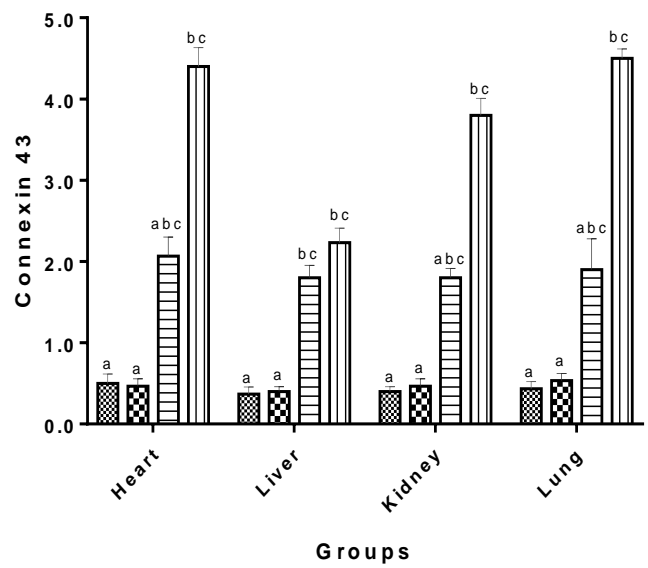

Figure 7. Effect of a single dose of doxorubicin $(20 \mathrm{mg} / \mathrm{kg}$ ) alone or combined with propolis $(100 \mathrm{mg} / \mathrm{kg})$ on A) Connexin 43, and B) Cytochrome-C immune expression in cardiac muscle, liver, kidney, and lung tissue sections. Groups: Control, Propolis, P+ DOX, DOX. Data are expressed as mean \pm SE $(n=10)$. a Significantly different from the DOX group at $p<0.05$, b Significantly different from the normal control group at $p<0.05$, and c Significantly different from the propolis group.

According to our knowledge, this study is the premier to determine the protective potentials of Australian propolis capsules in the market against DOX-induced multi-toxicity. These effects were confirmed according to several biochemical markers in addition to histopathological and immunohistochemical findings in the female rat model, exploring their mechanisms. Our results indicate that propolis appears to be rich in phenolic acids and flavonoids, which could explain its strong free radical scavenging activity in vitro, in accordance with other studies [15]. Most propolis samples from different geographical places were reported to have similar phenolic profiles, but the concentration of these compounds was different [16]. Among the identified compounds in propolis capsules in our study, rosmarinic acid, sinapic acid, and apigenin as the most abundant compounds. Also, cinnamic, ferulic, and caffeic acids, as well as quercetin and kaempferol, showed considerable amounts. These flavones and flavanols are effective flavonoids for protecting the body against reactive oxygen species (ROS). Both cinnamic and caffeic acid have shown antitumor effects in various cancer types [17]. Thus, the protective effects of propolis might be attributed to its antioxidant of these polyphenolic. We hypothesized that combining DOX with propolis would enhance the antitumor effect of DOX and prevent several damages caused by DOX.

DOX-induced cardiotoxicity, the main limitation to the clinical use of DOX as chemotherapy, was manifested in our study by increase the activities of serum diagnostic markers of heart damage as $\mathrm{CK}-\mathrm{MB}, \mathrm{CK}$, and LDH in addition to AST and ALT in the DOX group compared to control one. This cardiac damage may increase the permeability or rupture of the cell membrane, causing leakage of these cytosolic enzymes into the bloodstream and subsequent increasing their serum concentrations [18]. In line with these results, previous researchers found that DOX caused cardiac damage and loss of functional integrity based on significantly increased serum LDH, CK-MB, ALT, and AST levels [19]. Also, both TC and TG concentrations were elevated by DOX administration alone due to possible interference of DOX with metabolism or biosynthesis of lipids by decreasing the level of cytochrome P450. This leads to inhibit the activity of cholesterol 7-hydroxylase, the enzyme responsible for 
converting cholesterol to bile acids [20]. This abnormal lipid metabolism is frequently correlated with cardiovascular disease like atherosclerosis and others due to modifying the composition, structure, and stability of cellular membranes [21]. Our histopathological findings were consistent with our biochemical results, confirming DOX-induced cardiotoxicity also in the form of cardiac severe congestive hemorrhage, degenerative necrotic area, and fibrosis, revealing cardiomyopathy. On the contrary, the group pre-treated with propolis exhibited slight cardiac muscle necrosis and fibrotic blood vessels. In consistence with our biochemical results and with a previous study that showed the administration of propolis before isoproterenol significantly decreased the activities of CK-MB, LDH, AST, and ALT as well TG and TC levels in rats [21]. They revealed that the cardioprotective properties of Malaysian Propolis against isoproterenol-induced myocardial infarction through its direct cytotoxic radicalscavenging activities and lipid peroxidation inhibition. In the same line, a former study confirmed that the propolis cardio-protective effects are due to the antioxidant activity of its polyphenols and the generation of nitric oxide as a vasodilator besides the expression of genes that protect the cardiovascular system [22].

Our study also established DOX-induced liver toxicity based on increased activities of serum ALT, AST, and ALP in the untreated DOX group versus the control one. The increase in these indices of liver function may be due to an increase in the apoptotic processes in liver tissue as the hepatocytes are the main target for reactive oxygen attack [23]. Abnormal elevations in these hepato-specific enzymes in serum indicate hepatic cell damage, changing the permeability of the damaged cell membrane, thus releasing enzymes from the cells into the circulation [24]. Both AST and ALT enzymes are abundant in the heart and liver, thus their mild elevations have been associated with both liver injury and myocardial infarctions. In harmony with these results, [14] showed that Dox-induced elevated activities of AST and ALT may be attributed to the hepatocyte cell death caused by the oxidative stress leading to the leakage of hepatic cytosolic components as occurred with the heart. Conversely, propolis pretreatment significantly decreased serum ALT, AST, and ALP levels compared to the DOX group. This may be due to flavonoids' anti-inflammatory and antioxidant activity in propolis to capture and deactivate the free radicals that prevent liver damage, as stated before [17]. In the same line, there was a restoration of the nearly normal architecture of hepatic parenchyma in $(\mathrm{P}+\mathrm{DOX})$ group as propolis ameliorates most of the histopathological changes in the liver.

Data presented in this study demonstrated that DOX caused a rise in BUN, creatinine, and uric acid levels with hypoalbuminemia and hypoproteinemia. These results are consistent with previous findings where DOX-induced nephrotoxicity in normal rats [25]. They assumed that leakage occurred in the damaged kidney, allowing albumin to be excreted in the urine. On the other hand, the levels of serum BUN, creatinine, and uric acid in the propolis treated group was found to be significantly lower than in the DOX group, showing considerable protection to the kidney tissues. In accordance with the biochemical data, the histological abnormalities in the kidney showed obvious degenerative changes, glomerular tuft atrophy, and Bowman's capsule fibrosis that is also identical to what be found by previous results [26]. Also, DOXinduced renal injury might be initiated by an increased mitochondrial ROS production at first, then by an inflammatory response, as mentioned before [27]. In the same manner, DOX exhibited many histological alterations as inflammation and fibrosis in lung tissues. These results agreed with the previous study that showed the several side effects of DOX on lung tissue [28]. In comparison, others stated the chemoprotective effect of propolis against benzo $(\alpha)$ pyrene-induced lung carcinogenesis [29]. 
It is well documented that DOX as anthracycline has a quinone form converted into a semiquinone radical by reducing one electron. Then to regenerate this parent quinone again, molecular oxygen is reduced to superoxide anion, which then dissimulated into $\mathrm{H}_{2} \mathrm{O}_{2}$. These ROS causes oxidative stress by receiving electrons from the lipids in cell membranes, leading to lipid peroxidation and oxidant-induced cell injuries. Moreover, ROS can cause structural and functional damage to mitochondria, resulting in highly apoptotic features observed in our study [20]. Elevated MDA and decreased GSH in the cardiac and hepatic tissues of the DOX group in the current investigation revealed increased oxidative stress. Propolis treatment enhanced protection from oxidative stress by reducing both myocardial and hepatic contents of MDA while increasing their reduced GSH contents. The impact of DOX toxicity was greater in the liver tissue relative to the heart due to increased DOX concentration in the liver during the detoxification process. The same cause propolis treatment could not attenuate this concentrated DOX effect in the liver while in the heart. In agreement with our findings, a significant decrease in both superoxide dismutase activity and GSH content were observed with a significant increase in MDA content of cardiac tissue of rats exposed to DOX in previous work [18]. Furthermore, the hepatic MDA also was increased, while, GSH liver content was decreased significantly in DOX-induced hepatotoxicity in rats, as previously reported in several studies [30, 31]. However, in the $(\mathrm{P}+\mathrm{DOX})$ group, the cardiac contents of both MDA and GSH levels and the hepatic content of GSH were all found to be close to those observed in the control group. This can be explained that propolis can limit the free radicals production and improve the antioxidant defense system. Yet, the hepatic content of MDA showed no significant change versus the DOX group, matching with a previous study [3]. This may be attributed to propolis's dose-dependent inhibitory effects on lipid peroxidation in the liver, the main organ responsible organ for the breakdown and elimination of DOX.

Of note, besides oxidative stress, inflammation also has an essential role in the pathogenesis of DOX-induced multi-organ toxicities. Moreover, free radical's overproduction provokes more inflammatory mediators (NF- $\mathrm{\kappa B}$ and its subsequent pro-inflammatory cytokines generation), triggering many pathological alterations $[18,32]$. In the present study, it was found that DOX significantly increased IL-1 $\beta$, while propolis treatment inhibited its secretion. This led to the inhibition of a cascade that activated NF- $\kappa \mathrm{B}$, iNOS, and proinflammatory cytokines, thus the inflammatory response, as found in previous studies [2, 24]. According to the histological results, liver, heart, kidney, and lung damages and inflammation decreased by administering propolis in a similar way to the biochemical results. The pretreatment with propolis for 28 days before DOX causes significant amelioration in all previous markers compared to the DOX group, indicating that propolis helped maintain membrane integrity, restricting the leakage of the enzymes. Moreover, polyphenols presented in propolis capsules are important constitutive antioxidants that may protect against oxidative cardiac, hepatic, and renal injury. Also, these polyphenols inhibit many cellular enzymes like xanthine oxidase, lipoxygenase, and phospholipase $\mathrm{A}_{2}$, reducing LDL-peroxidation. In addition to their role as iron chelators, they also reduce the iron-dependent production of free radicals, increasing its scavenging activity and thus inhibiting the rate of lipid peroxidation [22]. Therefore, propolis has the potential to reduce coronary artery disease by norming lipids and preventing lipids oxidation [33]. In harmony with the present results, a preceding work showed that propolis anti-inflammatory effects were observed via reducing IL- $1 \beta$ expression along with COX-2, and IL-6 expression ROS and NO production [16]. Other conducted research 
yielded that the genes and protein expression levels of pro-inflammatory cytokines IL-1 $\beta$, IL6, and IL-8 decreased significantly after treatment with Brazilian propolis [34].

Similar protective effects against DOX-induced cardiotoxicity were further confirmed by the immunohistochemical results as the up-regulation of the mitochondrial $\mathrm{Cx} 43$ expression. Cx43 is a major gap-junction protein that mediates intercellular communication by supporting the passage of molecules and ions. It regulates electrical coupling, mitochondrial function, and synchronous contraction in cardiomyocytes. A previous study demonstrated that the alterations of $\mathrm{Cx} 43$ expression in DOX-induced several cardio-pathological conditions, such as heart failure due to mitochondrial dysfunction and oxidative stress. This $\mathrm{Cx} 43$ upregulation is an adaptive response to counteract the intracellular $\mathrm{Ca}^{2+}$ overload, ROS production, and the propagation of apoptotic signals induced by DOX [35]. Also, previous studies have exhibited the role of $\mathrm{Cx} 43$ in controlling apoptosis initiation by preventing the release of $\mathrm{Cyt}-\mathrm{C}$ from mitochondria into the cytosol and decreasing its availability to trigger off the caspase- 3 cascade and thus apoptosis [36]. In accordance with our results, a previous review stated that propolis flavonoids inhibit the release of Cyt-C from the mitochondria into the cytoplasm due to the inhibition of p53 expression [22]. Moreover, other researchers concluded that $\mathrm{Cx} 43$ inhibition protected against kidney injury through reducing ROS-mediated oxidative stress and inflammation reactions as well as regulated necroptosis [37]. Similarly, Cx43 downregulation protected the lung against lung vascular permeability, as stated before [38].

No significant change was detected between the healthy control animals and the propolis group, indicating that propolis did not induce any side effects. Moreover, previous studies showed that propolis did not alter the antitumor effect of DOX and even it possessed some anticancer activity [23].

\section{Conclusions}

These findings increased the possibility that propolis may be an adjuvant therapy, protecting organs from oxidative and apoptotic actions related to DOX, preventing its adverse effect. Further studies are needed to verify the role of propolis when used as curative management of DOX-induced multiple toxicities or to produce novel drug delivery strategies.

\section{Funding}

This research did not receive any specific grant from funding agencies in the public, commercial, or not-for-profit sectors.

\section{Acknowledgments}

This research has no acknowledgments.

\section{Conflicts of Interest}

The authors declare that there are no conflicts of interest.

\section{References}

1. Eleiwa, N.Z.H.; Galal, A.A.A.; Abd El-Aziz, R.M.; Hussin, E.M. Antioxidant activity of Spirulina platensis alleviates doxorubicin-induced oxidative stress and reprotoxicity in male rats. Orient Pharm Exp Med 2018, 18, 87-95, https://doi.org/10.1007/s13596-018-0314-1. 
2. Abdel-Daim, M.M.; kilany, O.E.; Khalifa, H.A.; Ahmed, A.A.M. Allicin ameliorates doxorubicin-induced cardiotoxicity in rats via suppression of oxidative stress, inflammation and apoptosis. Cancer Chemother Pharmacol 2017,80,745-753, https://doi.org/10.1007/s00280-017-3413-7.

3. Kaya, E.; Yılmaz, S.; Ceribasi, S. Protective role of propolis on low and high dose furan-induced hepatotoxicity and oxidative stress in rats. J Vet Res 2019, 63, 423-431, https://doi.org/10.2478/jvetres-20190054.

4. Rivero-Cruz, J.F.; Granados-Pineda, J.; Pedraza-Chaverri, J.; Pérez-Rojas, J.M.; Kumar-Passari, A.; DiazRuiz, G.; Rivero-Cruz, B.E. Phytochemical Constituents, Antioxidant, Cytotoxic, and Antimicrobial Activities of the Ethanolic Extract of Mexican Brown Propolis. Antioxidants(Basel) 2020, 9, 70, https://doi.org/10.3390/antiox9010070.

5. Ripari, N.; Sartori, A.A.; da Silva Honorio, M.; Conte, F.L.; Tasca, K.I.; Santiago, K.B.; Sforcin, J.M. Propolis antiviral and immunomodulatory activity: a review and perspectives for COVID-19 treatment, $J$ Pharm Pharmacol 2021, 73, 281-299, https://doi.org/10.1093/jpp/rgaa067.

6. Bozkuş, T.N.; Değer, O.; Yaşar, A. Chemical characterization of water and ethanolic extracts of Turkish propolis by HPLC-DAD and GC-MS. J Liq Chrom Relat Tech 2021, 44, 77-86, https://doi.org/10.1080/10826076.2021.1883648.

7. Marinova, G.; Batchvarov, V. Evaluation of the methods for determination of the free radical scavenging activity by DPPH. Bulg J Agric Sci 2011, 17, 11-24.

8. Bilgic, S.; Ozgocmen, M. The protective effect of misoprostol against doxorubicin induced liver injury. Biotech Histochem 2019, 94, 583-591, https://doi.org/10.1080/10520295.2019.1605457.

9. Karatas, F.; Karatepe, M.; Baysar, A. Determination of free malondialdehyde in human serum by highperformance liquid chromatography. Anal Biochem 2002, 311, 76-79, https://doi.org/10.1016/s00032697(02)00387-1.

10. Jayatilleke, E.; Shaw, S. A high-performance liquid chromatographic assay for reduced and oxidized glutathione in biological samples. Anal Biochem 1993, 214, 452-457, https://doi.org/10.1006/abio.1993.1522.

11. Bancroft, J.D.; Gamble, M.A. Theory and practice of histological techniques $6^{\text {th }}$ ed.; Churchil Livingstone: New York, London 2007, 165-175.

12. Rossé, T.; Olivier, R.; Monney, L.; Rager, M.; Conus, S.; Fellay, I.; Jansen, B. Borner, C. Bcl-2 prolongs cell survival after Bax-induced release of cytochrome C. Nature 1998, 391, 496-9, https://doi.org/10.1038/35160.

13. Levesque, R. SPSS programming and data management: a guide for SPSS and SAS users. $4^{\text {th }}$ edit. United States of America; 2007.

14. Sikandar, A.; Farhat, K.; Afzal, A.; Ajmal, K.; Laeeq, M.; Khokhar, A. Protective Effects Of Trimetazidine Against Doxorubicin-Induced Cardiotoxicity And Hepatotoxicity In Mice. J Ayub Med Coll Abbottabad 2020, 32, 304-309.

15. Woźniak, M.; Mrówczynska, L.; Waśkiewicz,A.; Rogoźinski,T.; Ratajczak, I. The role of seasonality on the chemical composition, antioxidant activity and cytotoxicity of Polish propolis in human erythrocytes. Rev Bras Farmacogn 2019, 29, 301-308, https://doi.org/10.1016/j.bjp.2019.02.002.

16. Asgharpour, F.; Moghadamnia, A.A.; Motallebnejad, M.; Nouri, H.R. Propolis attenuates lipopolysaccharideinduced inflammatory responses through intracellular ROS and NO levels along with downregulation of IL$1 \beta$ and IL-6 expressions in murine RAW 264.7 macrophages. J Food Biochem 2019, 43, e 12926, https://doi.org/10.1111/jfbc.12926.

17. Gal, A.F.; Stan, L.; Tăbăran, F.; Rugină, D.; Cătoi, A.F.; Andrei, S. Chemopreventive Effects of Propolis in the MNU-Induced Rat Mammary Tumor Model. Oxid Med Cell Longev 2020, 2020, https://doi.org/10.1155/2020/4014838.

18. Aziz, M.M.; Abd El Fattah, M.A.; Ahmed, K.A.; Sayed, H.M. Protective effects of olmesartan and L-carnitine on doxorubicin-induced cardiotoxicity in rats. Can J Physiol Pharmacol 2020, 98, 183-193, https://doi.org/10.1139/cjpp-2019-0299.

19. Bilgic, S.; Ozgocmen, M.; Ozer, M.K.; Asci, H. Misoprostol ameliorates doxorubicin induced cardiac damage by decreasing oxidative stress and apoptosis in rats. Biotech Histochem 2020, 95, 514-521, https://doi.org/10.1080/10520295.2020.1727013.

20. Mubarak, S.; Abdel Hamid, S.; Farrag, A.E.; Samir, N.; Hussein, J.S. Cardioprotective effect of date palm against doxorubicin-induced cardiotoxicity. Asian J Pharm Clin Res 2018, 11, 141-146, https://doi.org/10.22159/ajpcr.2018.v11i7.24453. 
21. Ahmed, R.; Tanvir, E.M.; Hossen, M.S.; Afroz, R. Ahmmed, I.; Rumpa, N.E.N.; Paul, S.; Gan, S.H.; Sulaiman, S.A; Khalil, M.I. Antioxidant properties and cardioprotective mechanism of Malaysian propolis in rats. Evidence-Based Complement Altern Med 2017, 2017, https://doi.org/10.1155/2017/5370545.

22. Braakhuis, A. Evidence on the health benefits of supplemental propolis. Nutrients 2019, 11, 2705, https://doi.org/10.3390/nu11112705.

23. Badr, M.O.T.; Edrees, N.M.M.; Abdallah, A.A.M.; Hashem, M.A.; El-Deen, N.A.M.N.; Neamat-Allah, A.N.F.; Ismai, H.T.H. Biochemical and Antioxidant effect of Doxorubicin hydrochloride and Propolis on N-methyl-N-nitrosourea (MNU) Induced Adenocarcinoma in Rats. Bull Univ Agric Sci Vet Med Cluj Napoca 2015, 72, 215-225, https://doi.org/10.15835/buasvmcn-vm:11208.

24. Kuzu, M.; Yıldırım, S.; Kandemir, F.M.; Küçükler, S.; Çağlayan, C.; Türk, E.; Dörtbudak, M.B. Protective effect of morin on doxorubicin-induced hepatorenal toxicity in rats. Chem Biol Interact 2019, 308, 89-100, https://doi.org/10.1016/j.cbi.2019.05.017.

25. Rajasekaran M. Nephroprotective effect of costus pictus extract against doxorubici-induced toxicity on wistar rat. Bangladesh J Pharmacol 2019, 14, 93-100, https://doi.org/10.3329/bjp.v14i2.39992.

26. Al-Karawi, M.A.M; Al-Shammari S.M.H.; Yaseen, M.M. Pathological study of kidney in male rats treated with doxorubicin in Diyala province. J Phys Conf Ser 2019, 1294, 062004, https://doi.org/10.1088/17426596/1294/6/062004.

27. Cardoso, D.F.; Coriolano, H.A.; Duarte, J.A. Regular voluntary running has favorable histological effects on doxorubicin-induced kidney toxicity in Wistar rats. Cell Tissue Res 2018, 374, 177-187, https://doi.org/10.1007/s00441-018-2840-z.

28. Hong, Y.; Che, S.; Hui, B.; Yang, Y.; Wang, X.; Zhang, X.; Qiang, Y.; Ma, H. Lung cancer therapy using doxorubicin and curcumin combination: Targeted prodrug based, $\mathrm{pH}$ sensitive nanomedicine. Biomed Pharmacother 2019, 112, 108614, https://doi.org/10.1016/j.biopha.2019.108614.

29. Brihoum, H.; Maiza, M.; Sahali, H.; Boulmeltout, M.; Barratt, G.; Benguedouar, L.; Mesbah, M. Dual effect of Algerian propolis on lung cancer: antitumor and chemopreventive effects involving antioxidant activity. Braz J Pharm Sci 2018, 54, e17396, https://doi.org/10.1590/s2175-97902018000117396.

30. Nagai, K.; Fukuno, S.; Oda, A.; Konishi, H. Protective effects of taurine on doxorubicin-induced acute hepatotoxicity through suppression of oxidative stress and apoptotic responses. Anticancer Drugs 2016, 27, 17-23, https://doi.org/10.1097/CAD.0000000000000299.

31. Omobowale, T.O.; Oyagbemi, A.A.; Ajufo, U.E.; Adejumobi, O.A.; Ola-Davies, O.E.; Adedapo, A.A.; Yakubu, M.A. Ameliorative Effect of Gallic Acid in Doxorubicin-Induced Hepatotoxicity in Wistar Rats Through Antioxidant Defense System. J Diet Suppl 2018, 15, 183-196, https://doi.org/10.1080/19390211.2017.1335822.

32. Wang, Z.Q.; Chen, M.T.; Zhang, R.; Zhang, Y.; Li, W.; Li, Y.G. Docosahexaenoic Acid Attenuates Doxorubicin-induced Cytotoxicity and Inflammation by Suppressing NF-kB/iNOS/NO Signaling Pathway Activation in H9C2 Cardiac Cells. J Cardiovasc Pharmacol 2016, 67, 283-289, https://doi.org/10.1097/FJC.0000000000000350.

33. El Menyiy, N.; Al-Wali, N.; El Ghouizi, A.; El-Guendouz, S.; Salom, K.; Lyoussi B. Potential therapeutic effect of Moroccan propolis in hyperglycemia , dyslipidemia , and hepatorenal dysfunction in diabetic rats. Iran J Basic Med Sci 2019, 22,1331-1339, https://doi.org/10.22038/ijbms.2019.33549.8004.

34. Yuan, X.; Wang, Y.; Shi, B.; Zhao, Y. Effect of propolis on preserving human periodontal ligament cells and regulating pro-inflammatory cytokines. Dent Traumatol 2018, 34, 245-253, https://doi.org/10.1111/edt.12411.

35. Pecoraro, M.; Pala, B.; Di Marcantonio, M.; Muraro, R.; Marzocco, S.; Pinto, A. Mincione, G.; Popolo, A. Doxorubicin-induced oxidative and nitrosative stress: Mitochondrial connexin 43 is at the crossroads. Int J Mol Med 2020, 46, 1197-1209, https://doi.org/10.3892/ijmm.2020.4669.

36. Pecoraro, M.; Sorrentino, R.; Franceschelli, S.; Del Pizzo, M.; Pinto, A.; Popolo, A. Doxorubicin-Mediated Cardiotoxicity: Role of Mitochondrial Connexin 43. Cardiovasc Toxicol 2015, 15, 366-376, https://doi.org/10.1007/s12012-014-9305-8.

37. Yuan, D.; Li, X.; Luo, C.; Li, X.; Cheng, N.; Ji, H.; Qiu, R.; Luo, G.; Chen, C.; Hei, Z. Inhibition of gap junction composed of $\mathrm{Cx} 43$ prevents against acute kidney injury following liver transplantation. Cell Death Dis 2019, 10, 1-18, https://doi.org/10.1038/s41419-019-1998-y.

38. O'Donnell, J.J.O.; Birukova, A.A.; Beyer, E.C.; Birukov, K.G. Gap Junction Protein Connexin43 Exacerbates Lung Vascular Permeability. PLOS One, 2014, 9, e100931, https://doi.org/10.1371/journal.pone.0100931. 